SUBJECT AREAS:

ELECTRONIC PROPERTIES

AND MATERIALS

NEMS

ACTUATORS

FERROELECTRICS AND MULTIFERROICS

Received

31 May 2013

Accepted

16 October 2013

Published

4 November 2013

Correspondence and requests for materials should be addressed to

Z.H.W. (zhihong.

wang@kaust.edu.sa)

or X.X.Z. (xixiang.

zhang@kaust.edu.sa)

\section{Bending-induced electromechanical coupling and large piezoelectric response in a micromachined diaphragm}

\author{
Zhihong Wang, Yingbang Yao, Xianbin Wang, Weisheng Yue, Longqing Chen \& Xi Xiang Zhang
}

Advanced Nanofabrication Core Lab, King Abdullah University of Science and Technology, Thuwal 23955-6900, Kingdom of Saudi Arabia.

We investigated the dependence of electromechanical coupling and the piezoelectric response of a micromachined $\mathrm{Pb}\left(\mathrm{Zr}_{0.52} \mathrm{Ti}_{0.48}\right) \mathrm{O}_{3}(\mathrm{PZT})$ diaphragm on its curvature by observing the impedance spectrum and central deflection responses to a small AC voltage. The curvature of the diaphragm was controlled by applying air pressure to its back. We found that a depolarized flat diaphragm does not initially exhibit electromechanical coupling or the piezoelectric response. However, upon the application of static air pressure to the diaphragm, both electromechanical coupling and the piezoelectric response can be induced in the originally depolarized diaphragm. The piezoelectric response increases as the curvature increases and a giant piezoelectric response can be obtained from a bent diaphragm. The obtained results clearly demonstrate that a high strain gradient in a diaphragm can polarize a PZT film through a flexoelectric effect, and that the induced piezoelectric response of the diaphragm can be controlled by adjusting its curvature.

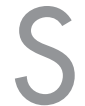

trong intrinsic coupling between electrical polarization and the uniform strain (piezoelectricity) has been demonstrated in thin films ${ }^{1-3}$. In comparison with this primary effect, flexoelectricity ${ }^{4,5}$, (coupling between electrical polarization and the strain gradient), a second-order effect, is generally trivially weak ${ }^{6}$. Approximately half a century ago, Bursian and Zaikovskii ${ }^{7}$ clearly demonstrated that in a cantilever composed of a layer of $\mathrm{BaTiO}_{3}$ thin film sandwiched between two electrodes, spontaneous polarization induced by an electric field may cause a distortion of the lattice which alters the film curvature. Their work also indicated that although flexoelectric effect exists in a wide range of dielectrics, it is likely to be large only in some ferroelectrics. However, the converse effect, i.e., the bending induced polarization, was not investigated in detail in their paper due to experimental difficulties. Over the last decade, L. E. Cross, a pioneer in experimentally assessing flexoelectric polarization, has shown with simple beam-bending experiments that strain gradients alone can induce noticeable changes in electrical polarization ${ }^{8-14}$, wherein the high dielectric constant accounts for the significant flexoelectric polarization. Although the debate on the absolute magnitude of the flexoelectric coefficients ${ }^{15-17}$ is still ongoing, recent reports reveal that giant flexoelectric polarization indeed exists on both the micro- and nanoscales ${ }^{18-22}$ due to the huge strain gradient on the order of $10^{4}$ to $10^{6} \mathrm{~m}^{-1}$ at film/substrate interface $e^{20,23}$ or in a nanoribbon ${ }^{24}$. Lu et al. ${ }^{18}$ were able to cause a reversal of electrical polarization in an ultrathin epitaxial $\mathrm{BaTiO}_{3}$ film with only the use of a strain gradient generated by the tip of a piezoresponse force microscope. Catalan et al. ${ }^{19}$ demonstrated that a horizontal strain gradient near the domain wall forces out-of-plane spontaneous polarization in the $\mathrm{c}$ domains to rotate away from the normal direction. In their work on wavy $\mathrm{Pb}(\mathrm{Zr}, \mathrm{Ti}) \mathrm{O}_{3}$ micro ribbons ${ }^{24}$, $\mathrm{Qi}$ et al. showed that local probing of buckled ribbons reveals a significant enhancement in the piezoelectric response due to the strain gradient that was found to be as high as $10^{4} \mathrm{~m}^{-1}$. Lee et al..$^{20}$ showed that the flexoelectric effect caused by strain gradients can create a strong imprint in uniaxial, perfectly oriented ferroelectric thin films. In addition, flexoelectricity due to substrate bending can also result in the imprint of polarization in polycrystalline ferroelectric films $\mathrm{s}^{21}$. Our recently published work ${ }^{22}$ has demonstrated that macroscopic strain gradients can induce substantial flexoelectric polarization in a bent $\mathrm{Pb}\left(\mathrm{Zr}_{0.52} \mathrm{Ti}_{0.48}\right) \mathrm{O}_{3}(\mathrm{PZT})$ diaphragm and that this giant flexoelectric polarization can reverse the remnant ferroelectric polarization in PZT thin films. Using a combination of atomistic and theoretical approaches, Majdoub et al. ${ }^{25}$ investigated the size dependent piezoelectric behavior of inhomogeneously strained nonpiezoelectric and piezoelectric nanostructures. They found that in materials that are intrinsically piezoelectric, flexoelectric and piezoelectric effects do not add linearly but instead exhibit a synergistic interaction. The latter leads to a strong size dependent enhancement of the effective piezoelectric coefficient. With the flexoelectric coefficient reported by Cross et al. ${ }^{13}$, they estimated that the effective 
piezoelectric constant of ferroelectric $\mathrm{BaTiO}_{3}$ films increase by $20 \%$ of its bulk value at a thickness of $8 \mu \mathrm{m}$ and exhibits a fourfold increase at a thickness of $1 \mu \mathrm{m}$. The aforementioned results suggest that strain gradients may be exploited for generating, stabilizing or switching electrical polarization in thin film systems to enhance piezoelectricity, which inspired us to investigate whether electromechanical coupling and the piezoelectric response of the diaphragm is tunable by varying its curvature. In this article, we used the impedance spectrum and central deflection in response to a small AC driving voltage to evaluate the electromechanical coupling and the piezoelectric response of the diaphragm. The curvature of the diaphragm was controlled by applying air pressure to the back of the diaphragm. The results revealed that a depolarized flat diaphragm does not initially exhibit electromechanical coupling or the piezoelectric response. However, when we applied a pressure to bend the diaphragm, we were able to induce electromechanical coupling and a giant piezoelectric response in the originally depolarized diaphragm. We found that the induced piezoelectric response was tunable by varying the curvature of the diaphragm. While efforts continue to be expended on developing new methods and mechanisms to enhance electromechanical responses via ferroelectrics ${ }^{26,27}$, the phenomenon observed here, i.e., that bending can significantly alter electromechanical responses, uncovers a new route to high performance piezoelectric MEMS devices through dynamic curvature (flexoelectric polarizaton) control.

\section{Results}

Controllable curvature of the diaphragm. We designed a special test setup (see Methods) to control the curvature of the PZT/Si diaphragm through a small pressure chamber connected to the back of the diaphragm. Figure 1 shows the effect of the chamber pressure on the surface profile of a PZT/Si diaphragm with very high tensile stress. Figure 1a shows the schematic multilayer structure of the diaphragm. Figure $1 \mathrm{~b}$ shows a measured three-dimensional profile of the bent diaphragm subjected to a positive chamber pressure of $2.70 \mathrm{kPa}$. In Figure 1c, we see that the original deflection height of the diaphragm is appsoximately $0.307 \mu \mathrm{m}$ when no air pressure is applied from the back chamber. Considering that the diameter of the diaphragm is $1.2 \mathrm{~mm}$, the original diaphragm profile is actually very flat. As expected, the convexity, and thus the curvature of the diaphragm can be deformed by adjusting the air pressure from the back chamber of the diaphragm. The deflection heights are $4.65 \mu \mathrm{m}$ at $2.70 \mathrm{kPa}$ and $-4.50 \mu \mathrm{m}$ at $-2.98 \mathrm{kPa}$. A positive pressure value means that the air pressure inside the chamber is higher than that of the outside ambient environment, and vice versa. It can be seen in Figure 1c that the positive pressure induces a higher central deflection than does the negative pressure of the same order. This is due to the asymmetric multilayer structure of the diaphragm (Figure 1a).

Microstructure of the PZT film and cross-sectional view of the diaphragm. Figure 2 shows the SEM images of the cross section of a fabricated diaphragm that is bent. The columnar structure of the PZT grain on the diaphragm can be clearly seen in the images. The composition of the PZT film is $\mathrm{Pb}\left(\mathrm{Zr}_{0.52} \mathrm{Ti}_{0.48}\right) \mathrm{O}_{3}$. It is located in the tetragonal phase side near the morphotropic phase boundary (MPB). We also performed an X-Ray diffraction (XRD) measurement (See Supplementary Figure S1) to understand the crystalline structures of the multilayer top surface of the fabricated diaphragm. The tested area covers diaphragm (Figure $2 \mathrm{~b}$ ) and the large, rigid supporting area (see Figure 2d), and it can be seen from Figure S1 (Supplementary Information) that the PZT layer consists of a randomly oriented polycrystalline tetragonal phase.

Bending induced electromechanical coupling. Resonance measurement is a standard method recommended by the IEEE standard ${ }^{28}$ to determine the electromechanical coupling properties of a piezoelectric material. During the measurement process, applying a small AC driving voltage to a polarized piezoelectric structure can excite the intrinsic electromechanical coupling of the structure. This coupling, as a measure of the polarization state, can be observed through a resonance peak in the impedance-frequency spectrum. On the other hand, a structure that is made from a nonpolarized material cannot respond to a small AC electric excitation because the structure does not possess electromechanical coupling. Therefore, in this paper, we used the resonance peak in the impedance spectrum to evaluate the electromechanical coupling behaviors of the diaphragm. The fundamental principle of this measurement method is described in Supplementary Information. Figure 3 shows the impedance spectra of the PZT/Si diaphragm at different air pressures. Shown in Figure $3 \mathrm{a}$ are the effects of the positive pressure on the impedance and phase spectra of the piezoelectric diaphragm. In its original flat state, i.e., when no pressure is applied, the diaphragm did not show a resonance peak in either the impedance spectrum or the phase-frequency spectrum, indicating that there is no electromechanical coupling in the diaphragm; in other words, the diaphragm is not piezoelectric. However, upon the application of a positive pressure of $1.33 \mathrm{kPa}$, a resonance peak emerged. Both the resonance peak height and the resonance frequency increased further with a higher positive air pressure of up to $2.70 \mathrm{kPa}$. Figure 4 shows the effective electromechanical coupling coefficients (See Supplementary Equation S1) calculated with the tested resonance and antiresonance frequencies in the impedance spectra. It is clear that the effective coupling coefficient increases as greater pressure is applied. In comparison with the variation of resonance and antiresonance peaks in the impedance spectra, the variation of the peaks in the phase spectra due to changes in pressure are more pronounced (See Figure 3a). Therefore, it is more straightforward to qualitatively indicate the electromechanical coupling in the phase spectra. Figure $3 \mathrm{~b}$ shows that the induced resonance peak in the phase spectra was reversible when the positive air pressure was lowered. However, a very weak peak, as indicated by the arrow in Figure 3b, remained when the positive pressure was fully removed. Similar phenomena were observed when negative pressures were applied (Figure 3c). That is, the induced resonance peak height and the resonance frequency increased with increasing negative pressure. The resonance peak was almost removed when the negative pressure was gradually reduced to zero from $-2.98 \mathrm{kPa}$ with only a very weak peak remaining.

The observed phenomena clearly indicate that the flat PZT/Si diaphragm initially does not exhibit electromechanical coupling behavior. There is no self-polarization in this highly stressed PZT film. This is probably because the high in-plane stress inhibits the formation of the out-of-plane polarization (c-domain). Upon the application of static air pressure to the diaphragm, the deflection of the diaphragm directly induced electromechanical coupling in the non-prepolarized PZT/Si diaphragm, and the electromechanical coupling was enhanced as the air pressure was increased. Therefore, strain gradient may play an important role in this phenomenon. Considering that the diaphragm consists of a PZT layer, it is reasonable to assume that strain gradient can induce electrical polarization in PZT films, thus endow the film with piezoelectric properties. However, it is currently unclear what the mechanism of the induced polarization is. From a material microstructure point of view, reorientation of grains and/or domains and phase transformation at MPB due to external poling voltage are the main causes of electrical polarization and may thus be the possible causes of the bending induced polarization. However, bending can only induce large tensile stress in diaphragm plane. Even if the large in-plane tensile stress could induce the reorientation of domains/grains or a new phase, the cdomains or the polarization axis of the induced new phase will rotate 

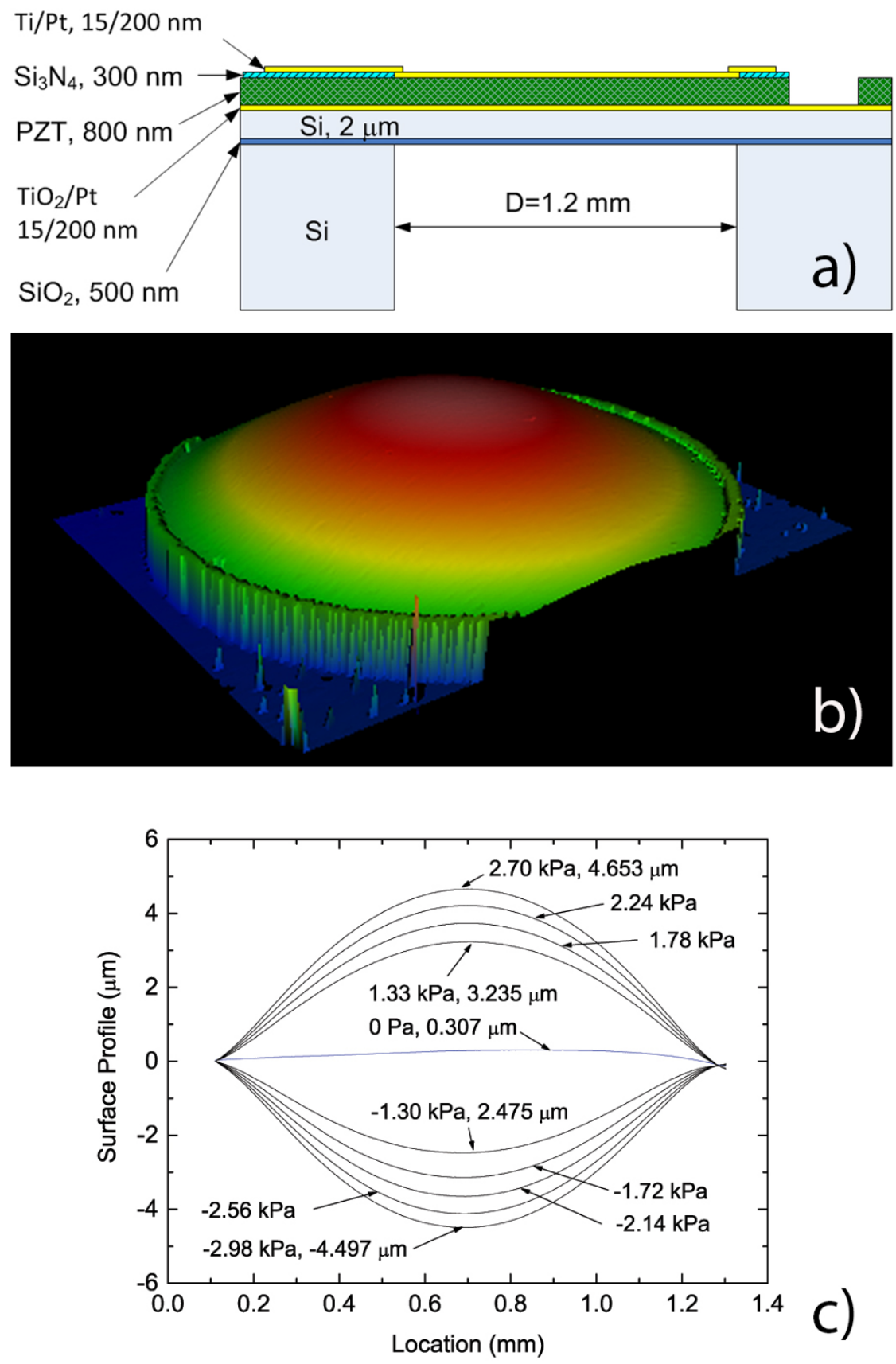

Figure $1 \mid$ Dependence of the surface profile of a PZT/Si diaphragm on the pressure difference between inside the chamber and the ambient atmosphere outside the chamber. (a) A schematic of the multilayer structure of the micromachined circular PZT/Si diaphragm. The diameter of the PZT/ Si diaphragm is $1.2 \mathrm{~mm}$. (b) This three-dimensional profile shows the curvature of the diaphragm at a positive chamber pressure. (c) A cross-sectional profile along the diaphragm's diameter at different chamber pressures. The positive values mean that the air pressure inside the chamber was higher than that of the ambient pressure outside the chamber.

away from the normal direction of the diaphragm so that the induced domain reorientation cannot enhance the out-of-plane polarization. Thus, the in-plane tensile stress induced reorientation of grains/ domains or phase transformation are unlikely to be the main causes of the bending induced out-of-plane polarization. In addition, it is not easy for the c-axis textured columnar grains of PZT (see Figure 2c) to rotate towards the in-plane direction. On the contrary, flexoelectric effect induces out-of-plane polarization, and is thus the most probable cause of the bending induced polarization.

The flexoelectric polarization should disappear when the strain gradient is removed, thus the very weak resonance peaks (Figures $3 \mathrm{~b}$ and $3 \mathrm{c}$ ) that are observed after the removal of applied pressure are caused by the residual strain in the diaphragm. Cross ${ }^{14}$ observed a similar residual strain in a PZT-5H specimen bar after a high stress four-point bend flexoelectric test. In comparison with the large remnant polarization after poling by an electric field in normal PZT films, the remnant component of the polarization from the residual strain gradient in highly stressed PZT films is very small.

Bending induced piezoelectric response in depolarized diaphragm. To confirm that the bending truly induces piezoelectric properties in PZT films, we also directly measured the piezoelectric response of the diaphragm under different levels of static pressure. The vibration amplitude at the center of the diaphragm was 

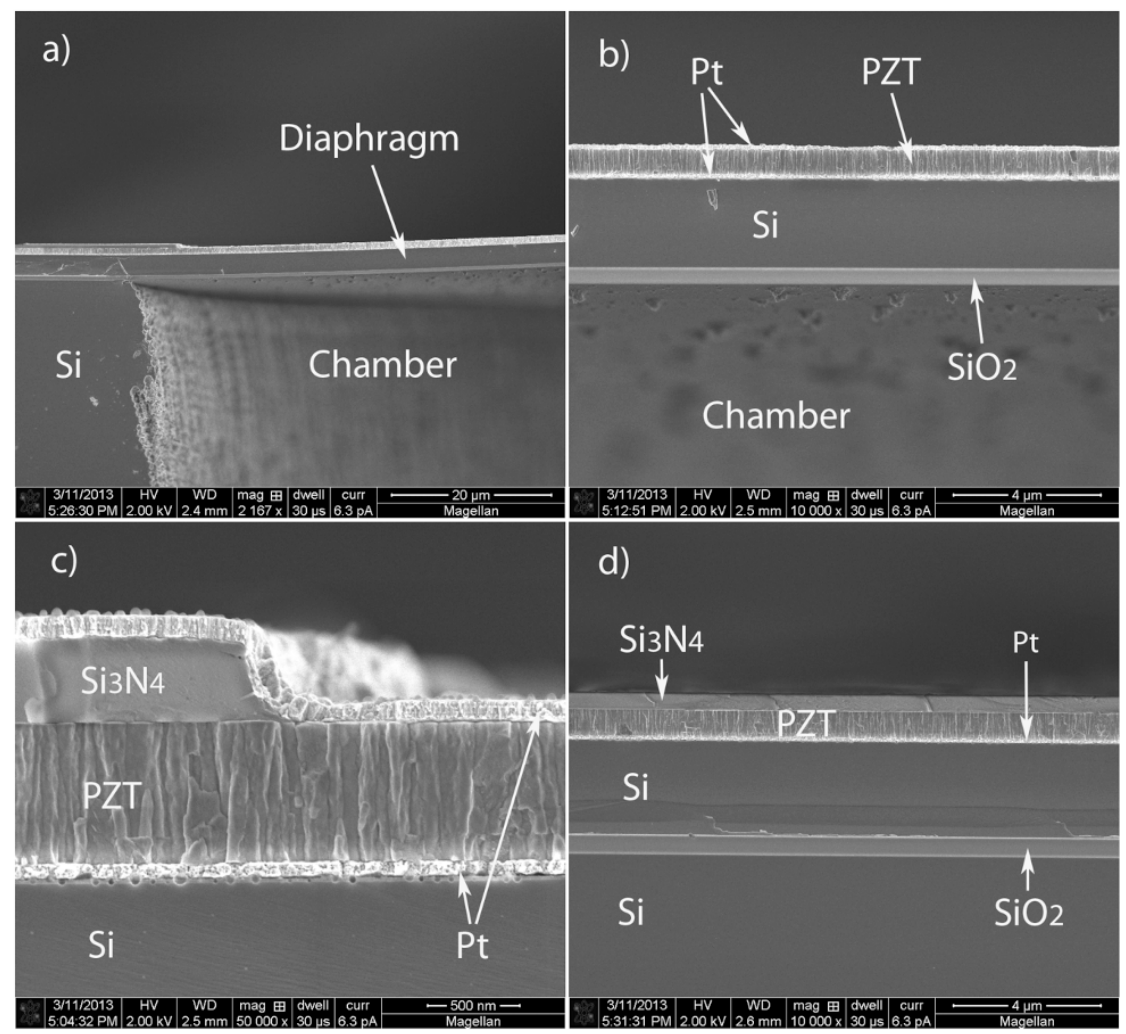

Figure $2 \mid$ Cross-sectional SEM images of a fabricated circular PZT/Si diaphragm. (a) Part of the released bent diaphragm together with the supporting Si substrate. (b) $\mathrm{Pt} / \mathrm{PZT} / \mathrm{Pt} / \mathrm{Si} / \mathrm{SiO}_{2}$ multilayer structure of the released $\mathrm{PZT} / \mathrm{Si}$ diaphragm. (c) Detailed cross-sectional view clearly shows the insulation $\mathrm{Si}_{3} \mathrm{~N}_{4}$ layer, top and bottom electrodes and the columnar grain structure of the PZT layer. (d) Multilayer structure far away from the diaphragm area. There is no Pt electrode on top of $\mathrm{Si}_{3} \mathrm{~N}_{4}$ layer and the silicon-on-insulator (SOI) substrate is clearly seen.

measured in response to a small AC driving voltage signal by using the standard single beam interferometer method for measuring the piezoelectric coefficient. The amplitude and frequency of the small AC driving signal were maintained at $0.5 \mathrm{~V}$ and $100 \mathrm{~Hz}$, respectively. The displacement amplitude-voltage coefficient ( $\mathrm{dv}$ coefficient), defined as the vibration amplitude of the diaphragm caused by the unit amplitude of the applied AC voltage, was used as a measure for evaluating the piezoelectric response of the diaphragm. Figure 5 shows the measured air pressure dependence of the $\mathrm{dv}$ coefficient. The $\mathrm{dv}$ coefficient was recorded in relation to a bipolar DC bias within $\pm 1 \mathrm{~V}$.

It can be seen in Figure 5 that the dv coefficient at the initial $0 \mathrm{~Pa}$ chamber pressure was linearly dependent on the DC bias within a relatively small range of $\pm 1 \mathrm{~V}$. It varied from around $0 \mathrm{~nm} / \mathrm{V}$ at $0 \mathrm{~V}$ to $-7.65 \mathrm{~nm} / \mathrm{V}$ at $+1 \mathrm{~V}$, returned to $0 \mathrm{~nm} / \mathrm{V}$ at $0 \mathrm{~V}$, and then increased to $+7.41 \mathrm{~nm} / \mathrm{V}$ at $-1 \mathrm{~V}$. It became zero again after removing the $-1 \mathrm{~V}$ bias. Normally, for a poled piezoelectric sample, the piezoelectric coefficient should remain a nonzero constant within a small bias range. Here, the zero dv coefficient at $0 \mathrm{~V}$ bias implies that the PZT film was initially at an depolarized state. Upon applying the bias, the $\pm 1 \mathrm{~V}$ bias-generated polarization was sufficient to exhibit perceptible piezoelectric responses $(-7.65 \mathrm{~nm} / \mathrm{V}$ at $+1 \mathrm{~V})$, and the $\mathrm{dv}$ coefficient only exhibited a slight hysteresis with the bias. The $\mathrm{dv}$ coefficient that linearly varied with the DC bias clearly demonstrates that it is not remnant polarization but electric field-induced polarization that causes this piezoelectric response. This electric fieldinduced polarization is normal and understandable. However, an abnormal phenomenon observed here is that, upon applying either a negative or positive external air pressure to bend the diaphragm, a nonzero piezoelectric response appears at $0 \mathrm{~V}$ bias. The bendinginduced $\mathrm{dv}$ coefficients at zero electric field are around $-17 \mathrm{~nm} / \mathrm{V}$ and $+19 \mathrm{~nm} / \mathrm{V}$ at $+1.33 \mathrm{kPa}$ and $-1.30 \mathrm{kPa}$, respectively. This observation is consistent with the results of electromechanical coupling shown in Figure 3, further confirming that bending the diaphragm can generate a piezoelectric response in the PZT film and that the piezoelectric response originates from the bending-induced flexoelectric polarization. Further increasing the air pressure (2.70 $\mathrm{kPa},-2.56 \mathrm{kPa}$ ) apparently does not increase the dv coefficient, which implies that the pressure-induced polarization reaches a saturation state at a certain air pressure. It is worth noting that when the air pressure is reduced from $3.64 \mathrm{kPa}$ and $-3.39 \mathrm{kPa}$ to zero, the $\mathrm{dv}$ coefficient almost falls to zero, with a small residue $(0.4 \mathrm{~nm} / \mathrm{V})$. This result is consistent with the observed relations between electromechanical coupling and air pressure (see Figure 3), i.e., the very small remnant component is also due to the residual strain gradient in the diaphragm.

To further investigate the bending-induced polarization of the film, we measured the $\mathrm{dv}$ coefficient hysteresis within a relatively large bias range of $\pm 6 \mathrm{~V}$. The small $\mathrm{AC}$ excitation signal we used during the test was $0.5 \mathrm{~V}$ at $1000 \mathrm{~Hz}$. We applied a single bipolar measurement cycle with the first half reaching $+6 \mathrm{~V}$ after applying a pre-polarization pulse of $-6 \mathrm{~V}$. We repeated the test at different levels of applied air pressure and the results are shown in Figure 6. In the initial state, where no external pressure is applied, the piezoelectric response ( $\mathrm{dv}$ coefficient) starts from zero at $0 \mathrm{~V}$ bias and returns to zero after reducing the bias from $-6 \mathrm{~V}$ to $0 \mathrm{~V}$. This result clearly shows that the selected $-6 \mathrm{~V}$ prepulse guarantees that the measurement always begins at the depolarization state. Several interesting phenomena can be clearly seen in Figure 6.

First, all the $\mathrm{dv}$-voltage hysteresis loops are negatively shifted, indicating an asymmetric piezoelectric response with respect to the bias voltage. It is interesting to note that all the curves tested at different pressures give a common bias of around $-2.7 \mathrm{~V}$, at which the $d v$ coefficients are zero. This result clearly indicates that an 
equivalent internal bias of $2.7 \mathrm{~V}$ should exist in the PZT film. Because of this equivalent internal bias, the remnant polarization after removing the $-6 \mathrm{~V}$ bias was fully counterbalanced. As a consequence, at all the applied chamber pressures, the remnant dv coefficient is almost zero after removing the $-6 \mathrm{~V}$ bias, whereas the remnant $d v$ coefficient is $-28.0 \mathrm{~nm} / \mathrm{V}$ at $0 \mathrm{~Pa}$ pressure after removing the $+6 \mathrm{~V}$ bias. It is clear that this considerable piezoelectric
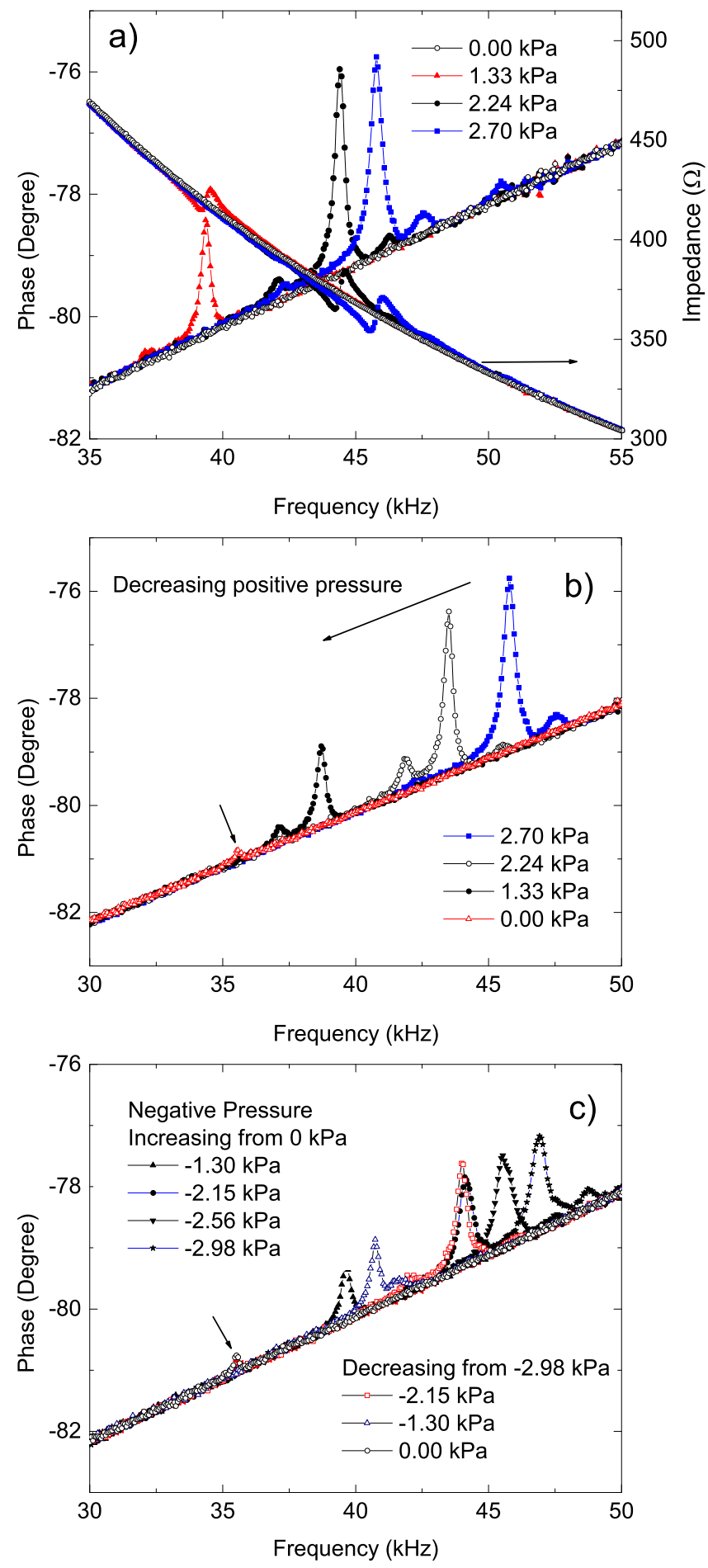

Figure 3 Static pressure-induced electromechanical coupling in a highly stressed PZT/Si diaphragm. (a) and (b) Dependence of the impedance/ phase-frequency spectra on positive pressures. (c) Dependence of the phase-frequency spectra on negative pressures.

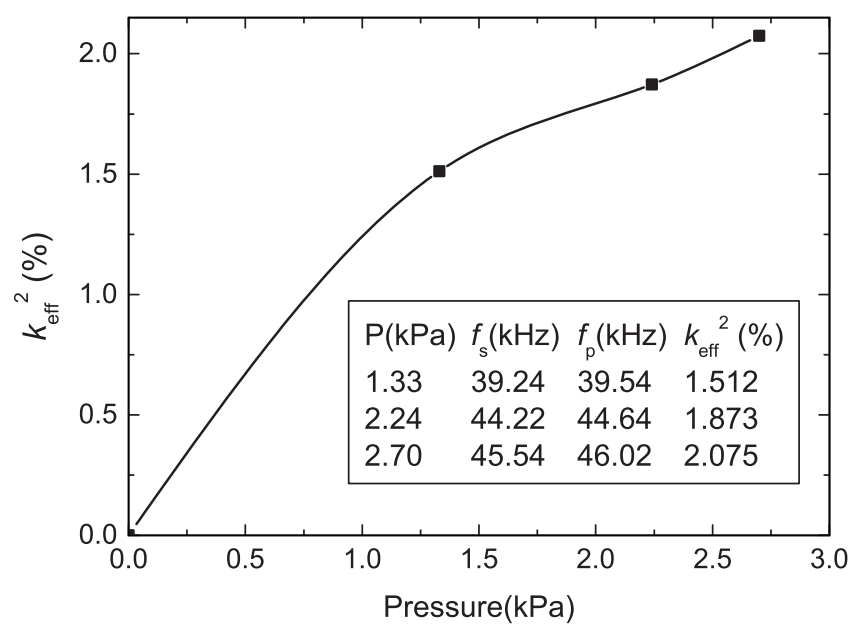

Figure $4 \mid$ Dependence of effective coupling coefficient on positive pressure. The coupling coefficient increase with the increase of the pressure. Inset table shows the resonance and anti-resonance frequencies obtained from impedance spectra as shown in Figure 3a.

response results from the remnant polarization generated by the concurrent action of both the external and internal electrical fields.

Second, although all the tests at different air pressures start from the same depolarization state, a nonzero initial $\mathrm{dv}$ coefficient at $0 \mathrm{~V}$ bias occurs upon application of an external air pressure. The absolute values of initial $\mathrm{dv}$ coefficients increase as the applied pressure increases irrespective of whether the pressure is negative or positive. This observation further implies that bending can induce polarization thus piezoresponse in PZT films. Although the bending-induced piezoresponse is small (the $\mathrm{dv}$ coefficients are around $-15.7 \mathrm{~nm} / \mathrm{V}$ at $3.17 \mathrm{kPa}$ and $15.6 \mathrm{~nm} / \mathrm{V}$ at $-3.39 \mathrm{kPa})$ in comparison with the $+6 \mathrm{~V}$ electrical bias-generated remnant piezoelectric response $(-28.0 \mathrm{~nm} / \mathrm{V}$ at $0 \mathrm{~Pa})$, it is in the same order of the magnitude. These experimental results demonstrate that bending-induced electrical polarization can generate a piezoelectric response at the same order of magnitude as that induced by an electric field, clearly showing that flexoelectric polarization plays an important role in micromachined PZT/Si diaphragms.

Third, bending can further enhance the electric field-induced piezoelectric response and generate a giant piezoelectric response in PZT diaphragms. The remnant piezoelectric response ( $\mathrm{dv}$

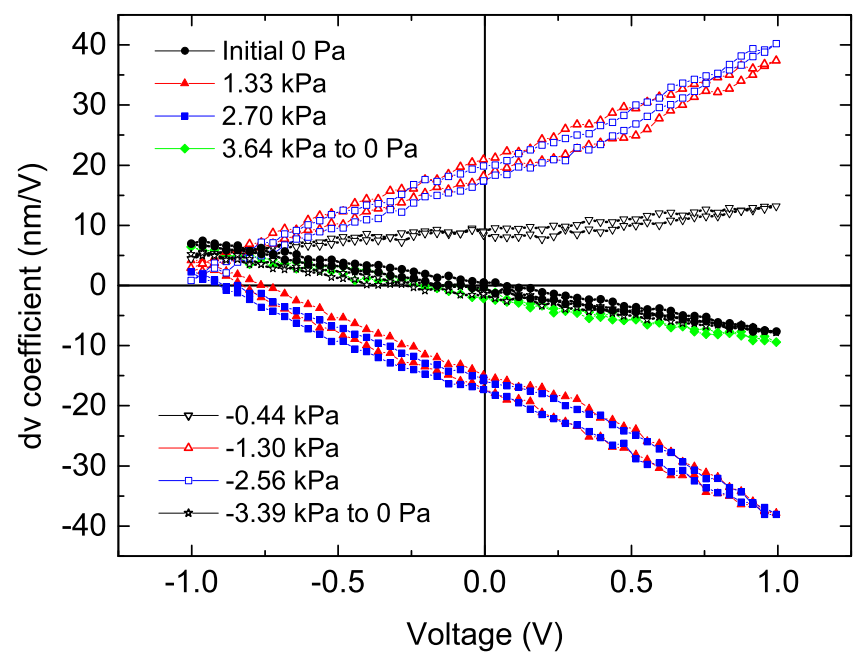

Figure 5 The dependence of the small-signal piezoelectric response of the PZT diaphragm on air pressure, starting from the depolarized state. 


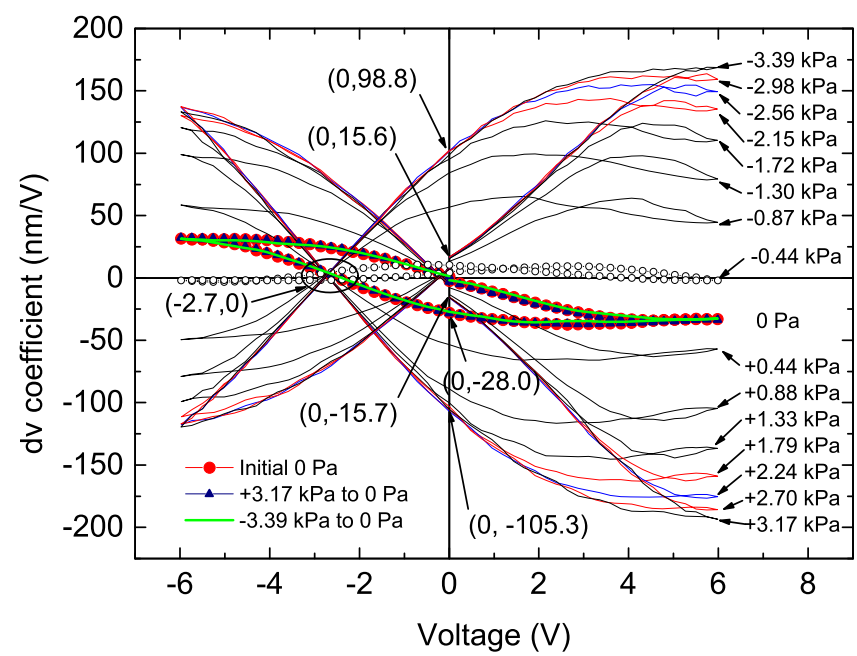

Figure 6 The dependence of the displacement voltage coefficient hysteresis curve of the PZT diaphragm on air pressure.

coefficient) increased as the air pressure increased after removing the $+6 \mathrm{~V}$ bias voltage. When a positive pressure was applied, the $\mathrm{dv}$ coefficient increased from $-28 \mathrm{~nm} / \mathrm{V}$ at $0 \mathrm{~Pa}$ to around $-105 \mathrm{~nm} /$ $\mathrm{V}$ at $3.17 \mathrm{kPa}$. Upon application of a negative pressure, the remnant $\mathrm{dv}$ coefficient first changed from negative to positive and then increased to $98.8 \mathrm{~nm} / \mathrm{V}$ at $-3.39 \mathrm{kPa}$. In addition to the enhanced piezoelectric response at a positive remnant polarization $(+\mathrm{Pr})$ state, we can clearly see in Figure 6 that the dv coefficient at the maximum bias of $\pm 6 \mathrm{~V}$ also increased with increased air pressure. At $+6 \mathrm{~V}$ bias, the $\mathrm{dv}$ coefficient was $-33.1 \mathrm{~nm} / \mathrm{V}$ at $0 \mathrm{~Pa}$ and increased to $-193.5 \mathrm{~nm} / \mathrm{V}$ and $168.9 \mathrm{~nm} / \mathrm{V}$ by respectively applying air pressures of $+3.17 \mathrm{kPa}$ and $-3.39 \mathrm{kPa}$. As a consequence of both the bending and applied bias, the dv coefficient of the PZT diaphragm can reach hundreds of nanometer per voltage, indicating that we can obtain high performance piezoelectric devices in a thin film system by utilizing flexoelectric polarization.

Curvature controlled piezoelectric response in a well-polarized diaphragm. To gain deeper understanding of how bending affects a polarized diaphragm, we examined the PZT/Si diaphragm after electrically poling the PZT layer by applying a $+20 \mathrm{~V}$ voltage at room temperature. Figure 7 shows the dependence of the piezoelectric response on the applied air pressure. A small AC signal of $0.5 \mathrm{~V}$ and $100 \mathrm{~Hz}$ was used to excite the vibration of the diaphragm. To avoid depolarization, the measurement was performed with a unipolar positive bias ranging from 0 to $3 \mathrm{~V}$. It is clear that during the tests, the bias apparently did not affect the $\mathrm{dv}$ coefficient. Figure $7 \mathrm{a}$ shows that the initial $\mathrm{dv}$ coefficient at $0 \mathrm{~Pa}$ pressure after poling remained almost constant within a $0-3 \mathrm{~V}$ range. It varied from -38.3 to $-34.7 \mathrm{~nm} / \mathrm{V}$ (see the red curve in Figure 7a). Again, the piezoelectric response of the diaphragm was enhanced substantially by the application of an external air pressure to bend the diaphragm. At $+3.17 \mathrm{kPa}$ pressure, the $\mathrm{dv}$ coefficient reached $-194 \mathrm{~nm} / \mathrm{V}$ (Figure $7 \mathrm{~b}$ ), around five times that at $0 \mathrm{~Pa}$. Upon removing the positive pressure, the remnant $\mathrm{dv}$ coefficient became larger (see the green curve in Figure 7a) due to the convex profile of the residue deformation or the increased flexoelectric polarization. Negative pressure changed the sign of the $\mathrm{dv}$ coefficient. At $-0.44 \mathrm{kPa}$, the dv coefficient varied from $+14 \mathrm{~nm} /$ $\mathrm{V}$ at $0 \mathrm{~V}$ to $+6 \mathrm{~nm} / \mathrm{V}$ at $3 \mathrm{~V}$. The $\mathrm{dv}$ coefficient increased as the negative pressure increased. It reached $+157 \mathrm{~nm} / \mathrm{V}$ at $-3.39 \mathrm{kPa}$ pressure (Figure $7 \mathrm{~b}$ ). After removing the negative pressure, the remnant $d v$ coefficient returned to its initial value (see the blue curve in Figure 7a).
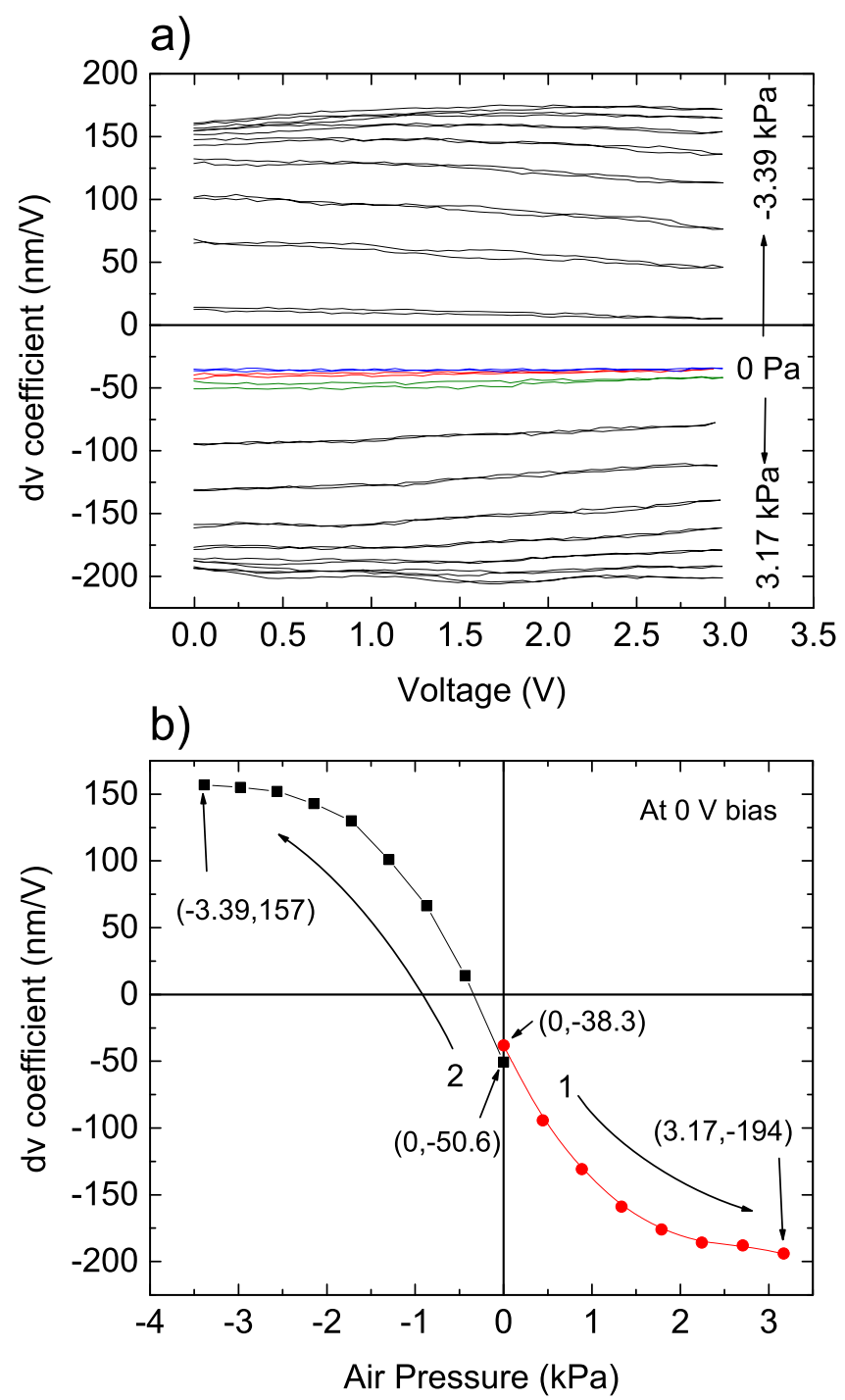

Figure $7 \mid$ Effect of the air pressure on the piezoelectric response of a poled PZT diaphragm. (a) The effect of the bias voltage on the dv coefficient at different air pressures. (b) The dependence of the small-signal piezoelectric response at remnant polarization state $($ at $0 \mathrm{~V})$ on the air pressure.

Zero and positive pressure resulted in negative $\mathrm{dv}$ coefficients and negative pressure resulted in positive $\mathrm{dv}$ coefficients, which can be explained by the direction of the remnant polarization and the curvature of the diaphragm. The profile measurement shows that the original diaphragm was not flat but was instead bent lightly upwards (see Figure 1c) when no pressure was applied. The application of positive pressure bent the diaphragm further upwards. In these cases, because the positive driving field was parallel to the remnant polarization, the increased lateral stress flattened the diaphragm and the $\mathrm{dv}$ coefficient became negative. On the contrary, if the application of negative pressure bent the diaphragm downwards, a positive driving field would bend the diaphragm upwards, resulting in a positive $\mathrm{dv}$ coefficient. It is evident from Figure $7 \mathrm{~b}$ that the $\mathrm{dv}$ coefficient can be significantly increased by increasing the air pressure from the back chamber. This giant piezoelectric response makes a bent diaphragm very promising in piezoelectric MEMS applications.

It is clear from Figure $7 \mathrm{~b}$ that the dv coefficient approaches a saturation state with the increase of applied pressure. Further increase of the pressure will not produce apparent enhancement of the piezoelectric displacement. This result reveals that for 
ferroelectric PZT diaphragm, although the flexoelectric effect and piezoelectric effect exhibit a synergistic interaction, the enhancement of the piezoelectric effect by flexoelectric polarization is limited. The saturated polarization that determined by microstructure of PZT film, and the nonlinear deflection-pressure relationship of the pressurized diaphragm might be the possible causes of this saturation effect, which needs to be further investigated.

Polarization-voltage loops. The polarization-voltage (P-V) hysteresis loops and the large-signal central deflection-voltage (D-V) butterfly curves were measured simultaneously on the same diaphragm. Figure 8 presents the results of two different tests. One starts from the + Pr state with the bipolar voltage cycle increasing to $-20 \mathrm{~V}$ first; while the other starts from the negative remnant polarization (-Pr) state with the voltage going to $+20 \mathrm{~V}$ first. Figure 8 shows that the two peak deflections in the $\mathrm{D}-\mathrm{V}$ butterfly curves occur at $\pm 5.33 \mathrm{~V}$, indicating perfect symmetry with respect to voltage. The slight difference in deflection heights at $\pm 5.33 \mathrm{~V}$ in the $\mathrm{D}$ - $\mathrm{V}$ curves, however, can be attributed to the asymmetric multilayer structure of the diaphragm (see Figure 1a). The PZT film on this relatively flat diaphragm also exhibits typically symmetric $\mathrm{P}-\mathrm{V}$ hysteresis loops without visible imprints. We can also see from Figure 8 that the relaxed remnant polarization, $P_{\text {rrel }}$, is very low in comparison with the remnant polarization, $P_{r}$. Symmetric P-E loops and the low relaxed remnant polarization may explain why self-polarization, which is present in bent diaphragms ${ }^{22}$, is not present in this flat $\mathrm{PZT} / \mathrm{Si}$ diaphragm. In the $\pm 20 \mathrm{~V}$ voltage range, the central deflection may vary by $-800 \mathrm{~nm}$. Considering that the initial deflection height of the diaphragm is around $307 \mathrm{~nm}$ (see Figure 1c), the $-800 \mathrm{~nm}$ displacement means that the convex profile of the diaphragm can be deformed to a concave profile by applying a large voltage signal, indicating that the diaphragm possesses the features of a flexural rigidity-dominated plate when no external pressure is applied. When the applied pressure is increased, the gradually increased tensile stress in the PZT film causes the diaphragm to behave like a tension-dominated membrane ${ }^{29}$. In this high pressure case, the small AC voltage signal during the $\mathrm{dv}$ coefficient test does not change the convexity of the diaphragm.

It is interesting to note that although the dv-voltage loops exhibit asymmetric features in the low voltage range, in the high voltage range, however, applying air pressure to bend the diaphragm does not affect the shape of the P-V loops (see Figure 9). One possible reason for this may be the limitations of the conventional virtual ground method we used for measuring the P-V loops. This method relies on the measurement of switching charges when the polarization is altered by an external field. The bending-induced static

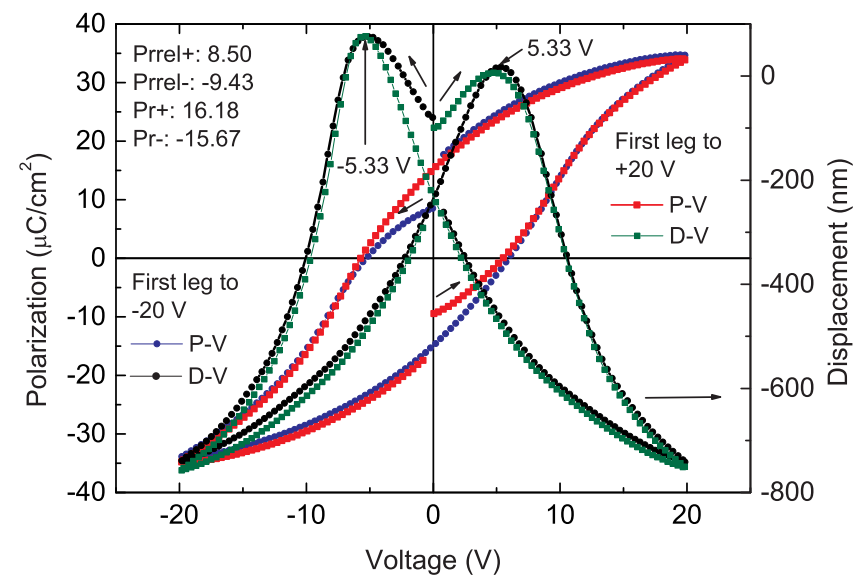

Figure 8 The large-signal central deflection-voltage butterfly curves in conjunction with the polarization-voltage hysteresis loops of the PZT/Si diaphragm when no air pressure was applied. flexoelectric polarization, which shifted the P-E loop along the $\mathrm{P}$ axis $^{22}$, could not be detected during the dynamic P-V loops test. In addition, if the maximum voltage during the $\mathrm{P}-\mathrm{V}$ test is high enough to reverse the flexoelectric polarization, there will be an identical saturated polarization for both the maximum negative and positive voltages. In this case, the shape of the P-V loop may be identical under different external air pressures. The flexoelectric effect contributes only to the relaxed remnant polarization. In fact, Figure 9 shows that the polarization at $\pm 20 \mathrm{~V}$ is around $35 \mu \mathrm{C} / \mathrm{cm}^{2}$, much higher than the flexoelectric polarization that could be obtained in this PZT/Si diaphragm, which was estimated at less than $2 \mu \mathrm{C} / \mathrm{cm}^{2}$ in our previous paper $^{22}$. In the low voltage range, however, the asymmetric P-E loop can be observed (see Figure 9) because the bending-induced polarization, together with the relaxed remnant polarization, can compete with the small electric field-induced polarization at the same order of magnitude. Even so, observing the P-E hysteresis loops using the conventional dynamic method is not a reliable way to investigate static flexoelecric polarization. On the other hand, the small-signal dv-voltage loops, which measure the piezoelectric response directly, can be employed as a criterion to assess the static polarization.

\section{Discussion}

We have demonstrated that thin film systems are ideal environments in which to capitalize on flexoelectric polarization. Electromechanical coupling and a giant piezoelectric response can be induced in a depolarized flat diaphragm by the application of air pressure which bends the diaphragm and generates flexoelectric polarization. The bending-induced giant piezoelectric response observed here opens a new way to exploit high-performance piezoelectric microelectromechnical devices by utilizing strain gradient-induced flexoelectric polarization.

\section{Methods}

Test setup to control the curvature of the PZT/Si diaphragm. Figure S2 (Supplementary Information) shows a picture of the test setup. A small pressure chamber was created in a stainless steel block. The chamber was connected to a syringe through a tube. A silicon chip with the PZT/Si diaphragm was placed on top of the chamber and sealed with paraffin. The pressure inside the chamber was controlled by a syringe pump (HARVARD/Apparatus) with an infusion or a withdrawal step of $25 \mu \mathrm{l}$. The pressure difference between the inside of the chamber and the ambient atmosphere outside the chamber was calculated by the volume change in both the chamber and the syringe by using the ideal gas law. The highest pressure difference during the test was around $\pm 3.5 \mathrm{kPa}$. This was high enough to bend the PZT diaphragm to an interesting curved shape.

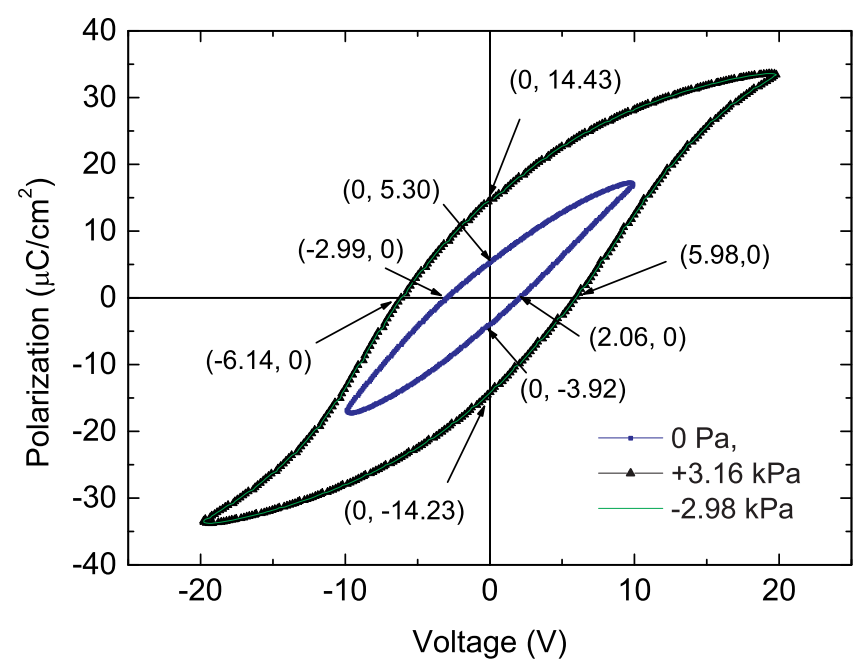

Figure $9 \mid$ P-V hysteresis loops at different external air pressures. The asymmetric feature in the low voltage range fades away in the high voltage range. The external pressure does not affect the shape of the P-V loop. 
Characterization methods. The surface profile of the diaphragm under different air pressures was measured by using an optical surface profiler (Zygo NewView 7300). An Agilent 4294A impedance analyzer was used to test the impedance spectrum of the diaphragm. To maintain consistency in the results, the high voltage port of the Agilent 4294A impedance analyzer was always connected to the top electrode of the diaphragm during the testing. The oscillation AC level of the activating voltage was $5 \mathrm{mV}$. The P-V hysteresis loops and piezoelectric response of the diaphragm were measured by an aixACCT TF Analyzer 2000E with a single beam laser interferometer. The vibration amplitude at the center of the diaphragm in response to a small AC driving voltage signal was measured using the single beam interferometer. The amplitude and frequency of the small AC driving signal was maintained at $0.5 \mathrm{~V}$ and $100 \mathrm{~Hz}$ unless otherwise denoted. The fabrication process of the PZT/Si diaphragm was described in detail in our previous paper ${ }^{22}$.

1. Choi, K. J. et al. Enhancement of ferroelectricity in strained $\mathrm{BaTiO}(3)$ thin films. Science 306, 1005-1009 (2004).

2. Haeni, J. H. et al. Room-temperature ferroelectricity in strained SrTiO3. Nature 430, 758-761 (2004).

3. Warusawithana, M. P. et al. A Ferroelectric Oxide Made Directly on Silicon. Science 324, 367-370 (2009).

4. Tagantsev, A. K. Piezoelectricity and Flexoelectricity in Crystalline Dielectrics. Phys. Rev. B 34, 5883-5889 (1986).

5. Tagantsev, A. K. Electric Polarization in Crystals and Its Response to Thermal and Elastic Perturbations. Phase Transit. 35, 119-203 (1991).

6. Gregg, J. M. Stressing Ferroelectrics. Science 336, 41-42 (2012).

7. Bursian, J. M. \& Zaikovskii, O. I. Changes in Curvature of a Ferroelectric Film Due to Polarization. Soviet Physics-Solid State 10, 1121-1124 (1968).

8. Ma, W. H. \& Cross, L. E. Observation of the flexoelectric effect in relaxor $\mathrm{Pb}(\mathrm{Mg} 1 / 3 \mathrm{Nb} 2 / 3) \mathrm{O}-3$ ceramics. Appl. Phys. Lett. 78, 2920-2921 (2001).

9. Ma, W. H. \& Cross, L. E. Large flexoelectric polarization in ceramic lead magnesium niobate. Appl. Phys. Lett. 79, 4420-4422 (2001).

10. Ma, W. H. \& Cross, L. E. Flexoelectric polarization of barium strontium titanate in the paraelectric state. Appl. Phys. Lett. 81, 3440-3442 (2002).

11. Ma, W. H. \& Cross, L. E. Strain-gradient-induced electric polarization in lead zirconate titanate ceramics. Appl. Phys. Lett. 82, 3293-3295 (2003).

12. Ma, W. H. \& Cross, L. E. Flexoelectric effect in ceramic lead zirconate titanate. Appl. Phys. Lett. 86, 072905 (2005).

13. Ma, W. H. \& Cross, L. E. Flexoelectricity of barium titanate. Appl. Phys. Lett. 88 232902 (2006)

14. Cross, L. E. Flexoelectric effects: Charge separation in insulating solids subjected to elastic strain gradients. J. Mater. Sci. 41, 53-63 (2006).

15. Zubko, P., Catalan, G., Buckley, A., Welche, P. R. L. \& Scott, J. F. Strain-gradientinduced polarization in SrTiO3 single crystals. Phys. Rev. Lett. 99, 167601 (2007).

16. Maranganti, R. \& Sharma, P. Atomistic determination of flexoelectric properties of crystalline dielectrics. Phys. Rev. B 80, 054109 (2009)

17. Hong, J. W., Catalan, G., Scott, J. F. \& Artacho, E. The flexoelectricity of barium and strontium titanates from first principles. J. Phys.-Condes. Matter 22, 112201 (2010).

18. Lu, H. et al. Mechanical Writing of Ferroelectric Polarization. Science 336, 59-61 (2012).

19. Catalan, G. et al. Flexoelectric rotation of polarization in ferroelectric thin films. Nature Materials 10, 963-967 (2011).
20. Lee, D. et al. Giant flexoelectric effect in ferroelectric epitaxial thin films. Phys. Rev. Lett. 107, 057602 (2011).

21. Gruverman, A. et al. Mechanical stress effect on imprint behavior of integrated ferroelectric capacitors. Appl. Phys. Lett. 83, 728-730 (2003).

22. Wang, Z. H. et al. Giant Flexoelectric Polarization in a Micromachined Ferroelectric Diaphragm. Advanced Functional Materials 23, 124-132 (2013).

23. Catalan, G., Noheda, B., McAneney, J., Sinnamon, L. J. \& Gregg, J. M. Strain gradients in epitaxial ferroelectrics. Phys. Rev. B 72, 020102 (2005).

24. Qi, Y. et al. Enhanced Piezoelectricity and Stretchability in Energy Harvesting Devices Fabricated from Buckled PZT Ribbons. Nano Lett. 11, 1331-1336 (2011).

25. Majdoub, M. S., Sharma, P. \& Cagin, T. Enhanced Size-dependent Piezoelectricity and Elasticity in Nanostructures Due to the Flexoelectric Effect. Phys. Rev. B 77, Artn 125424 (2008)

26. Baek, S. H. et al. Giant Piezoelectricity on Si for Hyperactive MEMS. Science 334, 958-961 (2011).

27. Sluka, T., Tagantsev, A. K., Damjanovic, D., Gureev, M. \& Setter, N. Enhanced electromechanical response of ferroelectrics due to charged domain walls. Nat. Commun. 3, 748, Doi 10.1038/Ncomms1751 (2012).

28. IEEE Standard on Piezoelectricity, ANSI/IEEE Standard 176-1987, New York: IEEE, Inc., page 49, 1987.

29. Olfatnia, M., Xu, T., Ong, L. S., Miao, J. M. \& Wang, Z. H. Investigation of residual stress and its effects on the vibrational characteristics of piezoelectric-based multilayered microdiaphragms. J Micromech. Microeng. 20, 015007 (2010).

\section{Acknowledgements}

The authors would like to thank Professors Weiguang Zhu and Xi Yao for valuable discussions, Mr. Jun Li for taking the SEM images, Dr. Liang Li for carrying out XRD test, Messrs. Jian Ren and Xiaodong Xu for help with the design and machining of the pressure chamber, and Dr. Virginia Unkefer for improving the English.

\section{Author contributions}

Z.W. conceived and designed the experiments, carried out the measurements, and prepared the manuscript. Y.Y., X.W., W.Y. and L.C. contributed to the interpretation of the expremental data. X.X.Z. coordinated the project and co-wrote the manuscript. All authors discussed the results and commented on the manuscript.

\section{Additional information}

Supplementary information accompanies this paper at http://www.nature.com/ scientificreports

Competing financial interests: The authors declare no competing financial interests.

How to cite this article: Wang, Z. et al. Bending-induced electromechanical coupling and large piezoelectric response in a micromachined diaphragm. Sci. Rep. 3, 3127; DOI:10.1038/ srep03127 (2013)

(c) (1) (-) This work is licensed under a Creative Commons Attribution-

BY NC SA NonCommercial-ShareAlike 3.0 Unported license. To view a copy of this license, visit http://creativecommons.org/licenses/by-nc-sa/3.0 